\title{
Deportivización y proceso de secularización en el Uruguay (1870-1930)
}

http://dx.doi.org/10.11606/1807-5509202000034nesp039

Paula MALAN ${ }^{*}$

*Instituto Superior

de Educación

Física, Universidad

de la República,

Montevideo, Uruguay.

\section{Resumen}

El objetivo de este trabajo es explorar las afinidades entre el proceso de deportivización y el proceso de secularización en el Uruguay, durante el período comprendido entre 1870 y 1930, a partir de la relación establecida entre el Estado, las minorias protestantes y la Asociación Cristiana de Jóvenes (ACJ). El proceso de institucionalización del deporte en Uruguay estuvo ligado al accionar de misioneros protestantes norteamericanos que llegaron a la región, desde finales del siglo XIX, para proyectar la instalación de las primeras Asociaciones Cristianas de Jóvenes (ACJs) en Sudamérica. En 1919, Uruguay se constituye en el primer país laico de Sudamérica, al producirse la separación definitiva entre el Estado y la Religión Católica. En 1922, Montevideo fue elegida por la Confederación Sudamericana de ACJs como localidad para instalar el primer Instituto Técnico Sudamericano de ACJs, donde se formarían los primeros secretarios y directores de educación física del continente. La hipótesis principal presentada en este trabajo es que la práctica deportiva envolvería un programa religioso, poco reconocido en los estudios históricos a nivel nacional, el cual permitiria comprender el modo en que el deporte jugó un rol clave en el proceso de secularización en el Uruguay. La doctrina de la Cristiandad Muscular, en vínculo con la ACJ y las minorias protestantes, y por medio de la práctica deportiva, habría aportado algunos de los rasgos que hicieran posible la configuración de un nuevo ethos o modelo de sujeto laico en Uruguay, durante el periodo 1870-1930. Desde una perspectiva histórica que recoge las afectaciones del análisis del discurso francés, se exploran fuentes documentales pertenecientes a archivos privados y al Centro de Memoria de Educación Física (CEMEF) en Uruguay.

Palabras clave: Estado Uruguayo; Deporte; Asociación Cristiana de Jóvenes; Minorías protestantes; Laicidad.

\section{Introducción}

El mismo día y año en que se decretaba la ley que suprime toda enseñanza y práctica religiosa de las Escuelas Públicas del Estado Uruguayo, se fundaba la ACJ de Montevideo. Luego de la puesta en marcha de la Reforma Constitucional (1919) que decretó la separación definitiva del Estado de la Iglesia Católica, se instaló en la capital del país el Instituto Técnico Sudamericano de ACJs (1922) para la formación de secretarios y directores de educación física a nivel continental.

A lo largo de este ensayo nos preguntamos el porqué de estas coincidencias: ¿cuál es la relación entre la ACJ y el proceso de laicidad y secularización en el Uruguay?, ¿por qué el movimiento asociacionista eligió, dentro de los países del continente, a Uruguay, como centro de difusión y traducción de esta formación en educación física que traía como elemento distintivo y revolucionario la enseñanza del deporte? ¿Cuál es la relación entre deporte y matriz secular?

Un conjunto de investigaciones a nivel mundial han referido al movimiento de la ACJ como clave para entender el proceso de incorporación de las prácticas deportivas modernas y el desarrollo de una moralidad cristiana secular en la sociedad inglesa y canadiense, desde mediados del siglo XIX hacia la primera mitad del siglo $\mathrm{XX}^{1-3}$. En el continente americano, varios estudios señalan la influencia de la ACJ en la 
consolidación del campo de la Educación Física ${ }^{4-8}$.

En Uruguay, los estudios señalan la influencia del movimiento de ACJs norteamericano en el proceso gestacional de la Educación Física en el país, como un suceso mayor y más importante que en otros países del continente ${ }^{9-14}$. En trabajos anteriores ${ }^{15-17}$, se analiza el proceso de incorporación de prácticas deportivas en las colonias valdenses del Uruguay durante la primera mitad del siglo XX, en vínculo con la ACJ y con otras denominaciones protestantes.

Si bien la secularización ha sido ampliamente abordada en el campo de la historiografía uruguaya ${ }^{a}$, la vinculación de este proceso con las minorías protestantes $^{\mathrm{b}}$ y la $\mathrm{ACJ}$, aún es un tema poco explorado.

A partir de la revisión de algunas fuentes documentales, y desde una perspectiva histórica que incorpora las afectaciones del análisis del discurso francés, este ensayo esboza algunas líneas teóricas en el camino de comenzar a comprender las afinidades entre la ACJ y el proceso de secularización en el Uruguay. En este recorrido, nos detendremos en una articulación fundamental: el deporte y las minorias protestantes de la mano de la doctrina de la cristiandad muscular.

La hipótesis principal que orienta este trabajo es que el proceso de deportivización jugó un rol determinante en la conformación del sujeto moderno laico en el Uruguay (1870-1930), a partir del lazo establecido entre el Estado, las minorías protestantes y la ACJ, bajo el influjo de la doctrina de la Cristiandad Muscular.

Tomando distancia de una perspectiva de oposición dicotómica entre secularidad y religiosidad, este trabajo propone una relectura de las nociones de secularización y deportivización, identificando en la confluencia de ambos procesos, aquellos rasgos de religiosidad, que durante el período de modernización en el Uruguay, habrían permitido la conformación de una matriz ciudadana deportiva y secular.

\section{Proceso de secularización e institucionalización de las prácticas deportivas en el Uruguay (1870-1930)}

Entre finales del siglo XIX y principios del XX ${ }^{\mathrm{d}}$ se produjo el proceso de modernización en el Uruguay. Este se caracterizó por un período de profundas transformaciones estructurales que desembocaron en la afirmación de un estado moderno laico.

Bajo un modelo de "ciudadanía hiperintegradora", que sostuvo como condición indispensable para la integración política y social el abandono de las identidades de origen (religiosas, étnicas, lingüísticas, culturales, etc.), cuatro grandes procesos se encaminaron hacia la conformación de la nueva "identidad nacional" de los uruguayos: la reforma escolar vareliana, la nacionalización de los inmigrantes, la medicalización, y la radicalización de la secularización ${ }^{18}$ (p. 35).

La historiografía nacional ${ }^{e}$ coincide en señalar como etapa medular del proceso de secularización en el Uruguay, la primera modernización capitalista en el país (1870-1930). Durante este período, diversas medidas estatales fueron limitando la influencia eclesiástica sobre la sociedad civil hasta llegar a la separación definitiva entre el Estado y la Religión Católica. Entre ellas: la municipalización de los cementerios (1861), el decreto-ley de Educación Común (1877), la creación del Registro de Estado Civil (1879), el Matrimonio Civil Obligatorio y la Ley de Conventos $(1885)^{19}$. En 1918, el Proyecto de Reforma Constitucional estableció la separación de la Iglesia Católica del Estado y la conformación de la actual República Oriental del Uruguay. Este proyecto entraría en vigencia a partir de 1919.

En este período, varios acontecimientos ilustrarían el proceso sistemático a través del cual la educación física y el deporte se fueron instalando como elementos claves de las políticas educativas a nivel nacional. Entre ellos: la inclusión de la gimnasia y de los juegos al aire libre en la Reforma Escolar propuesta por Varela (1877), el Proyecto de Ley para la Creación de los Juegos Atléticos Anuales (1906), la creación de la Comisión Nacional de Educación Física (CNEF) (1911), la creación del Instituto Técnico de la Confederación Sudamericana de ACJs (1922), la aprobación del primer Plan de acción de Educación Física a nivel nacional (1923), la construcción del estadio nacional de fútbol y primer mundial de fútbol en Uruguay $(1930)^{\mathrm{f}}$.

Hasta ahora hemos transitado por el discurso de modernización que ha comandado la escritura de la historiografía uruguaya. En este, la modernización se presenta como aquel proceso que tuvo sus inicios en la segunda mitad del siglo XIX, y que estuvo pautado por una continua y progresiva laicización, secularización y racionalización; y cuyo corolario sería la articulación de una estructura política democrática y participativa que posibilitó el nacimiento de la República. A este discurso de modernización se acoplaría el discurso de institucionalización de la educación física y del deporte.

Esta construcción del relato histórico nacional, 
sería semejante a la noción de secularización manejada a finales del siglo. Según TAYLOR ${ }^{20}$, hacia los años sesenta del siglo XX, una serie de teólogos, filósofos y sociólogos, defensores y críticos de la religión, llegaron al consenso de que la secularización es inseparable del proceso de modernización, y que cuando nos referimos a esta estamos hablando del proceso a través del cual la sociedad y la cultura dejan de estar controladas por las instituciones y los símbolos religiosos. Dentro de esta matriz discursiva descriptiva y normativa, la desaparición de la religión es considerada como señal de superación, de progreso humano, como si se tratase, en un imaginario de maduración, de un movimiento que va de lo infantil a lo maduro, de la superstición a la ilustración. Esta matriz se constituye sobre la base de una lógica de oposición dicotómica exclusivista que polariza, por un lado, lo religioso, y por el otro, lo secular, que aparecen como contrarios irreconciliables. Sin embargo, a principios del siglo XXI, se plantearían indicios de crisis de esta matriz interpretativa:

Aquellos pronósticos ingenuos respecto a una continua e ineluctable expansión de la secularización - según las formas y el tono "moderno" y "progresista" del liberalismo del siglo XIX - parecen haberse derrumbado. Lo que emerge en su lugar no parece sencillo de describir y calificar. Los procesos que se anotan resultan múltiples y a menudo contradictorios: surgimiento de nuevos movimientos y prácticas de carácter religioso; desinstitucionalización y diseminación de "lo religioso" en la sociedad; irrupción de una "nebulosa místico-esotérica" que se consagra en una suerte de "religión a la carte", fuertemente individual y móvil; crecimiento en distintas partes del mundo de movimientos extremistas e integristas con fondo religioso; reformulación de las modalidades de relación entre religión y política; etc. ${ }^{21}$ (p. 18) .

El propio tono de esta cita da a entender de qué modo este "resurgir de lo religioso" constituye un desenlace inesperado del proceso de secularización, en el marco de una matriz interpretativa dicotómica. Nos preguntarnos: ¿¿de qué modo estos indicios de crisis de la matriz interpretativa sobre el proceso de secularización repercutieron en el relato histórico nacional? ¿Hasta qué punto es o ha sido reconocida esta crisis en el marco de la historiografía uruguaya? Hacia finales del siglo XX y principios del XXI, la historiografía nacional habría convergido en la necesidad de rediscutir el proceso de secularización en el Uruguay. Los estudios ${ }^{\mathrm{h}}$ sobre la temática en esta época advirtieron acerca de la recolocación general de manifestaciones religiosas en el espacio público, y sobre la necesidad de redefinir las fronteras entre lo público y lo privado. Los indicios de esta crisis, parecieran no haber pasado desapercibidos para los historiadores, sin, que, al parecer, hayan en sus estudios cambiado de matriz interpretativa.

Se hace necesario entonces revisar la noción de secularización en el contexto de la modernidad occidental, encontrando las claves de una nueva matriz de interpretación. TAYLOR ${ }^{20}$ nos da una pista por dónde seguir:

La secularidad es un fenómeno religioso, dado que, de hecho, la religión, tal como se ha desarrollado en Occidente, siempre ha dado refugio a la secularidad, la secularidad prolonga, encubiertamente, un programa religioso. En otras palabras, secularidad y religión son coemergentes y codependientes $^{20}$ (p. 161).

Este es el punto de partida de la discusión que nos interesa plantear en este trabajo. Consideramos necesario indagar en la historia nacional, en particular, en la historia de la educación física, indicios de que el componente religioso, de que rasgos de religiosidad, podrían haber cumplido, desde antes ya, un papel determinante en la conformación de los sujetos modernos, que no pudimos percibir antes porque, de algún modo, estábamos capturados, en nuestra comprensión de los hechos, por el modelo dicotómico de interpretación sobre la secularización. Se trataría, entonces, de reevaluar, de redescubrir la presencia de estos elementos religiosos, incluir en la escena de los procesos de subjetivación también estos otros elementos que, de alguna manera, coadyuvaron, y sobredeterminaron, de modo tan fuerte, el modo de ser sujetos modernos.

Recordemos el supuesto que guía este trabajo: el proceso de secularización en el Uruguay estuvo intimamente ligado al proceso de deportivización.

Pasemos a preguntarnos ahora por la noción de deportivización. Siguiendo a Elias ${ }^{22}$, la deportivización puede ser entendida como aquel proceso a través del cual algunas formas de pasatiempos practicadas por caballeros y aristócratas en Inglaterra durante el siglo XVIII -las que empleaban la fuerza y la agilidad muscular, como ser la caza, el boxeo, las carreras y algunos juegos de pelota-, pasaron a adoptar un código de reglas más definidas, que limitaron la violencia y regularon la tensión entre el esfuerzo físico y el goce del resultado. 
Esta transformación de los pasatiempos derivó en la denominación de estos como deportes, término técnico que pasaría a diferenciarlos de otras formas recreativas.

Sin embargo, este proceso de deportivización desarrollado por Elias no puede llegar a comprenderse, sin incluir en la escena del relato el proceso de parlamentarización. Tanto el deporte como el parlamento fueron parte del mismo cambio de estructura de poder y de hábitos sociales que se experimentó en Inglaterra durante el siglo XVIII, a partir de la lucha de poder establecida entre el rey - la aristocracia - y los grandes terratenientes. El régimen parlamentario accedió, en este período, a que ambos grupos pasaran a estar representados en el parlamento, permitiendo que las diferencias de intereses se resolvieran en público y por medios no violentos, de acuerdo a reglas acordadas y aceptadas por ambas partes previamente. Se conjuga de este modo la afinidad entre ambos procesos: "la 'parlamentarización' de las clases hacendadas de Inglaterra tuvo su equivalente en la 'deportivización' de sus pasatiempos" 23 (p. 48)

El término deportivización también habría pasado a denominar el proceso a través del cual algunos de los deportes creados en Inglaterra durante los siglos XVIII y XIX se exportaron al resto de las sociedades, principalmente entre 1850 y 1950, difundiéndose con el término inglés sports ${ }^{22,24}$.

Luego de este recorrido por las nociones de secularización y deportivización, nos interesa hacer una salvedad que tiene que ver con la propia construcción de nuestro problema de investigación. Consideramos que un elemento clave en la articulación de estos procesos de modernización, secularización y deportivización, fue el componente religioso que sostuvo la práctica deportiva.

GUTTMAN ${ }^{25}$ plantea como primera característica distintiva de los deportes modernos la secularidad, así como la posibilidad de pensar a estos como una religión secular. ¿Pero qué significa religión secular en el contexto moderno?

Siguiendo a TAYLOR ${ }^{20}$, esta religión secular o componente de religiosidad en la modernidad, no habría sido genérica, sino que estaría estructuralmente atravesada por el protestantismo:

La secularidad es un fenómeno religioso que se origina cuando la tradición judeocristiana da lugar al protestantismo. Es más, no es una exageración insistir en que no sólo el mundo moderno, sino también el posmoderno, comenzó, de hecho, con la revolución protestante del siglo XVI.
(...) la globalización tiene una dimensión religiosa, no reconocida, que no refleja una espiritualidad genérica, sino que es protestante hasta la médula ${ }^{20}$ (p. 22-3).

Ahora bien, ¿cómo advertir entonces la relación ente secularidad, modernidad, protestantismo y deporte? Para comprender esta relación es necesario introducir la doctrina de la Cristiandad Muscular. Para ello, debemos retrotraernos a la primera mitad del siglo XVIII, cuando se produce en Europa el movimiento de avivamiento espiritual y religioso denominado Risveglio o Primer Gran Despertar, que se inició en Inglaterra y rápidamente se difundió por el resto de la Europa protestante y la América británica. Este movimiento prerromántico de renovación evangélica, con énfasis en el desarrollo de una espiritualidad interior e individual, habría dado origen al Metodismo ${ }^{26}$.

A inicios del siglo XIX se produciría el Segundo Gran Despertar. En continuidad con el anterior, y con un nuevo componente romántico, este movimiento acentúa el rasgo individual de la experiencia religiosa y profundiza la actividad misionera a través de un mayor compromiso con las obras de evangelización y expansión hacia el mundo secular. Este movimiento es el que habría provocado en Inglaterra la conformación de la Young Men's Christian Association (YMCA) (1844) y la renovación de la Iglesia Anglicana.

El movimiento de renovación de la Iglesia Anglicana habría sido impulsado por integrantes de una corriente político-religiosa que surgió a mediados del siglo XIX y que se denominó Socialismo Cristiano ${ }^{i}$ - quienes fueron también los creadores de la Teología de la Encarnación - y que promoverían el movimiento o doctrina que más nos interesa aquí, la denominada Muscular Christianity. Esta doctrina impulsaría la conformación de un nuevo ethos ${ }^{k}$ burgués, asociado a una moralidad individual cristiana y masculina, sobre la base de una condición física fuerte y saludable, y virtudes tales como el esfuerzo, el sacrificio, la competitividad y el coraje ${ }^{27}$ (p. 1). El enunciado de la Cristiandad Muscular insistiría en el "significado del alma encarnada, y la bondad del athleticism ${ }^{1}$ y la fuerza física en la formación del carácter" ${ }^{28}$ (p. 4). El gentleman-amateur inglés, a través de la práctica de los deportes modernos, encarnaría el ideal de este nuevo ethos cristiano y muscular ${ }^{29}$ - como veíamos anteriormente en la descripción de ELIAs $^{22}$ sobre la génesis del deporte.

Este ideal en la formación del carácter del joven, 
sobre la base de un equilibrio espiritual, mental y corporal, va a convergir con el movimiento asociacionista ${ }^{\mathrm{m}}$ cuando este incluya en su programa al deporte ${ }^{\text {n: }}$

\begin{abstract}
En sus actividades, la Asociación puede aparecer a una buena parte del público, quizás a la mayoría, como una institución de carácter primordialmente deportivo. Los que están más vinculados con ella saben que ella persigue también un fin intelectualmente educacional (...) Pero lo que deseen conocer bien su carácter deben saber que tanto sus actividades deportivas como su labor educacional tienen una finalidad moral y están inspiradas por un ideal religioso $0^{30}$ (p. 7-13).
\end{abstract}

De este modo, a la causa evangélica se le suma la causa deportiva, o más que eso, la práctica deportiva pasa a constituir el soporte material de esta nueva ideología religiosa, de tendencia dominante, la Cristiandad Muscular ${ }^{16}$, que se difundirá por medio de la ACJ en todo el continente sudamericano:

(...) la Asociación Cristiana de Jóvenes se halla en condiciones excepcionales para realizar esta gran obra de difundir el espíritu religioso cristiano entre la juventud. No tan sólo por el gran contacto que ya se haya establecido entre nuestras asociaciones y nutridos grupos de jóvenes de toda índole en la Argentina, Brasil, Chile, Perú y Uruguay, sino por la gran independencia de pensamiento y de acción que le da su posición de institución interconfesional y aconfesional, basada en el más amplio y comprensivo concepto del cristianismo" ${ }^{30}$ (p. 7-13).

Esta posición discursiva "interconfesional $y$ aconfesional" de la ACJ se muestra cercana a una posición secular, en la que se unen todas las religiones a la vez que ninguna. Esta condensación antinómica estaría representada de forma más cabal por el meta-enunciado de la Cristiandad Muscular ${ }^{\circ}$, el que conjuga en su enunciación términos pertenecientes a dos formaciones discursivas opuestas. Por un lado, "Cristiandad", término perteneciente a la discursividad religiosa. Por otro lado, "Muscular", proveniente de una discursividad científica, biológica, fisiológica. PÊCHEux ${ }^{31}$ es mediante este proceso de desplazamiento y sustitución entre formaciones discursivas -en este caso a través de la inclusión del término aconfesional dentro de una formación discursiva religiosa-, que las referencias discursivas se construyen y se desplazan históricamente, constituyendo una nueva estructura semántica de significado paradojal, como lo es el sentido "interconfesional y aconfesional". Esta posición discursiva antinómica habría permitido a la ACJ colocarse en una posición privilegiada de mediación, en el diálogo entre el Estado laico Uruguayo y las diferentes confesiones religiosas.

\section{Estado Uruguayo: indicios de una matriz secular deportiva}

Volvamos ahora al Estado Uruguayo, en búsqueda de aquellos indicios de que un componente religioso interconfesional y aconfesional, albergado en la doctrina de la Cristiandad Muscular, habría impulsado, desde finales del siglo XIX, la consolidación del proceso de secularización a través del proceso de deportivización.

José Pedro Varela (1845-1879), conocido como el gran reformador de la escuela uruguaya, viajó por Europa y Estados Unidos entre 1867 y 1868, introduciendo a su vuelta en el país, la corriente positivista de procedencia anglosajona principalmente spenceriana y darwiniana, así como el liberalismo racionalista que se impondría como corriente de pensamiento frente a la Iglesia Católica ${ }^{9}$ (p. 65). En 1876, Varela asumió la Dirección de la Instrucción Pública en el Uruguay, donde presentó el proyecto de Ley de Educación Común, proponiendo una enseñanza escolar laica, gratuita y obligatoria. Este proyecto fue aprobado y convertido en ley el 24 de agosto de 1877 (Decreto Ley de Educación Común). Sin embargo, el decreto de laicidad no se hará oficial hasta el 6 de abril de 1909 , fecha que coincide con la fundación de la ACJ de Montevideo.

Durante las últimas décadas del siglo XIX, mientras "se procesaba el enfrentamiento entre la Iglesia Católica y el Estado por la ocupación del espacio público, los protestantes comenzaban a tener una presencia destacada en el país"32 (p. 109). Hacia finales de aquel siglo y comienzos del $\mathrm{XX}$, los protestantes hicieron alianza con aquellas corrientes de pensamiento que promovieron el proceso de secularización y privatización de lo religioso - espiritualismo y positivismo -, como ocurrió en la mayoría de los países latinoamericanos. Son numerosas las publicaciones en los periódicos metodistas El Evangelista, El Atayala y El Estandarte Evangélico, y en el periódico valdense La Unión Valdense donde se apoya la política del Batllismo con respecto a la Iglesia Católica, afirmando que las funciones del Gobierno no son las religiosas. Desde su fundación en Montevideo, la ACJ estuvo claramente ligada a los grupos protestantes ${ }^{\mathrm{p}}$. La 
Iglesia Metodista habría ejercido un rol catalizador en la relación de la ACJ con los demás grupos minoritarios protestantes.

En 1906, la Cámara de Representantes discutía el primer plan racional de educación física a implantarse en el país:

En los tiempos que corren no se concibe ya un plan de educación racional que no comprenda al mismo tiempo el desarrollo educacional en su triple orientación: la educación intelectual, fisica y moral (...). De ahí el rol complejo que ejerce la educación física, de ahí que su acción no se limite exclusivamente a la vigorización del cuerpo, sino que actuando como actúa sobre todos los órganos y obrando sobre el cerebro, el órgano noble por excelencia en la especie, ejerza una influencia poderosa sobre el desarrollo de la mentalidad del individuo, sobre la educación de los sentimientos, sobre la formación del carácter y de la voluntad y hasta sobre la moralidad y la evolución estética de las razas $y$ de las sociedad, eso sin tener en cuenta todavía la honda repercusión que todos estos hechos realizan en el terreno económico, político y social ${ }^{33}$ (p. 7).

El elemento más sustantivo que se incorporó en el proyecto de este plan, fue el deporte. Véase el mensaje del presidente de la República - José Batlle y Ordoñez ${ }^{q}$ - que acompañó la consecución de este plan:

Un escritor francés que había residido largo tiempo en Londres, decía que a su regreso a Francia, la multitud le había parecido menos bella que la multitud inglesa. Allá, agrega, el hombre es esbelto, fino, de una real elegancia, y esa superioridad parece responder a dos causas: alimentación más racional, y práctica diaria de los deportes. Estimulemos, pues, los deportes, recordando que influyen eficazmente en la mayor salud del pueblo, y son además, una escuela insustituible de voluntad y de ánimo ${ }^{34}$ (p. 4).

En el marco de este discurso modernizador, el proceso de "medicalización de la moral" - señalado por BARRÁN $\mathrm{N}^{35}$ - podría entenderse como un proceso de "deportivización de la moral".

En 1912, llegó a Uruguay Jess T. Hopkins, egresado del International Y.M.C.A. College de Springfield, para ocupar el cargo de director físico en la ACJ de Montevideo.

Entre 1913 y 1918, Hopkins ocupó el cargo de director general de la CNEFr. Desde su rol colaboraría activamente en el desarrollo y la ejecución del primer Plan de Acción Nacional de Educación Física. Este plan impulsará especialmente la práctica de los deportes a través de la instalación de "Plazas vecinales de cultura física" en todo el país ${ }^{33}$. Siguiendo a SCarlato ${ }^{36}$, las plazas vecinales de cultura física, durante las primeras décadas del siglo XX, habrían oficiado como verdaderos "templos laicos" en el espacio público montevideano, en donde a través de la difusión de nuevas prácticas y saberes sobre el cuerpo se expandiría la nueva "religión civil".

Ya instalado en Montevideo, Hopkins escribió una carta al director de Springfield Y.M.C.A. College, advirtiendo sobre la posición influyente de Uruguay en el continente y sobre el modo en que la $\mathrm{ACJ}$ va tejiendo el vínculo con el gobierno uruguayo:

Conard, nuestro secretario y un graduado de la Universidad de Illinois, y Monteverde su asociado, un uruguayo muy influyente, han sentado las bases para un maravilloso trabajo de la asociación en este pequeńo pero importante país. Hablando de este país, no puedo describirlo mejor que como lo hizo el historiador sudamericano, Dawson: "el pequeño y valiente Uruguay ejerce y siempre ejercerá una influencia en los asuntos sudamericanos enormemente desproporcionada en relación a su tamaño" En poco más de tres años, estos dos hombres han construido una membresía de 750 de los cuales 6/7 son uruguayos, tienen en su comisión algunos de los hombres más influyentes en el cuerpo diplomático. Monteverde pasó una hora hoy con el presidente, describiéndole nuestro trabajo, el presidente odia todo lo que sabe a cristianismo, pero al final, cuando terminó, se fue con el ejecutivo alabando nuestro trabajo ${ }^{37}$ (p. 1).

Uruguay, primer país en Sudamérica en lograr la separación definitiva de la Iglesia Católica del Estado (1918), habría sido escogido por la ACJ en este período como lugar de traducción de un movimiento deportivo secular que se dirigía al resto de los países del continente con sus diversas realidades religiosas, y ciertamente, con mayor presencia de la Iglesia Católica (como ser Brasil y Argentina en este período) en el afán de instalar un nuevo orden corporal racional, disciplinar, individual, cristiano y muscular:

Muy honda es la huella que la Asociación viene dejando en la vida e ideales deportivos de nuestro Continente. (...) Ella también ha fomentado las plazas de deportes para niños en varias repúblicas sudamericanas. En dos de estas, por lo menos, hombres preparados en institutos de la Asociación, han sido nombrados por los gobiernos respectivos Directores Nacionales de Educación Física ${ }^{38}$ (p. 9-10). 
En 1922, la Federación Sudamericana de ACJs crea en Montevideo el Instituto Técnico Sudamericano de las $A C J s$, donde se formarían los secretarios y directores de educación física de toda América Latina. Este instituto recibió líderes provenientes de Argentina, Brasil, Chile, Perú, México y Uruguay. Los dos primeros años eran realizados en las ACJs locales y los dos últimos en la sede de Montevideo. La formación impartida por este instituto fue la primera formación sistemática y de larga duración en el campo profesional de la Educación Física a nivel nacional y sudamericano. A nivel nacional los semilleros de estudiantes de este Instituto fueron los grupos de jóvenes protestantes.

En relación a lo que significó la instalación de este instituto a nivel sudamericano, HopkINs ${ }^{39}$ señala:

Después de muchos años de estudio y ante una necesidad
sin cesar creciente de preparación y entrenamiento de líderes
nacionales, se estableció en el año 1922 el Instituto Técnico
con sus escuelas locales (...). Este esfuerzo representa, sin
duda, el acontecimiento más importante producido en el
desarrollo de nuestra obra en Sudamérica ${ }^{39}$ (p. 52).

Vemos de qué modo esta nueva determinación discursiva en relación a la educación del cuerpo - la que identificamos en trabajos anteriores ${ }^{15-17}$ como Cristiandad Muscular - se infunde por medio de la ACJ y a través del deporte, desde Uruguay hacia el resto del continente.

\section{Consideraciones finales}

A lo largo de este ensayo fuimos tejiendo algunos de los argumentos que permiten sostener la hipótesis de este proceso de indagación.

Tomando distancia de una perspectiva de oposición dicotómica entre secularidad y religiosidad, este trabajo propuso una relectura de las nociones de secularización y deportivización, identificando en la confluencia de ambos procesos, aquellos rasgos de religiosidad, que durante el proceso de modernización en el Uruguay, habrían permitido la conformación de una matriz ciudadana deportiva y secular. La posición discursiva "interconfesional y aconfesional" de la ACJ, parecería favorecer su ubicación en posición privilegiada en la mediación entre el Estado laico Uruguayo y las diferentes confesiones religiosas.

Uno de los principales argumentos elaborados en este trabajo fue que la presencia de un elemento religioso, poco reconocido en los estudios históricos sobre el deporte en el Uruguay, permitiría explicar el modo en que el proceso de deportivización jugó un rol clave en el proceso de secularización en el Uruguay. Este elemento, mediante la práctica deportiva, habría aportado algunos de los rasgos que hicieran posible la configuración de un nuevo ethos o modelo de sujeto laico en el Uruguay, durante el período 1870-1930. Este elemento religioso correspondería a la doctrina de la Cristiandad Muscular, la que habría permitido consolidar, a través de la práctica deportiva, un lazo estrecho entre la ACJ, el Estado Uruguayo y las minorías protestantes.

La instalación del Instituto Técnico Sudamericano de las ACJs en Uruguay, hecho que es considerado por los dirigentes de la ACJ como el acontecimiento más importante producido por la obra asociacionista en Sudamérica en las primeras décadas del siglo XX, aún es un nudo a desentrañar. Para ello será necesario poder explorar el archivo aún no creado de la ACJ en Uruguay.

Los primeros indicios puestos a funcionar en este trabajo mostrarían una relación estrecha entre los procesos de laicidad y secularización en el Uruguay, y entre la ACJ y las minorías protestantes. La hipótesis principal que guía este trabajo es que la práctica deportiva - bajo el influjo de la doctrina de la Cristiandad Muscular- habría constituido el soporte material de la causa secular que se difundiría por medio de la ACJ desde Uruguay al resto del continente. Será necesario ahondar en el diálogo mantenido entre las diferentes denominaciones protestantes, en el mundo católico y no cristiano, así como en el diálogo que sostuvo el Estado con los diferentes grupos religiosos; para identificar las especificidades y localizaciones de los procesos de resistencia e incorporación de la práctica deportiva en las diferentes comunidades. 


\section{Notas}

a. Dentro de los estudios más sobresalientes en esta temática encontramos los trabajos de Zubillga y Cayota ${ }^{41}$, BARRÁN ${ }^{35,42,44}$, Da Silveira ${ }^{45}$, Caetano y Geymonat ${ }^{18}$, Da Costa ${ }^{46,47}$.

b. Caetano y Geymonat ${ }^{18}$ en su libro "La secularización uruguaya (1859-1919) Catolicismo y privatización de lo religioso. Tomo 1", plantean el proyecto de un Tomo 2 para profundizar sobre el lugar de los protestantes en este proceso, pero este no se logra llevar a cabo.

c. El protestantismo ingresó al Uruguay a través de las llamadas iglesias étnicas (ingleses anglicanos, valdenses y reformados suizos). Recién en 1860 se establece un protestantismo de tipo misionero con la presencia de metodistas episcopales de raíz norteamericana32 (p.100-104),

d. Zubillga y CAYOta ${ }^{41}$, BarráN ${ }^{42-44}$, Sansón ${ }^{19}{ }^{49}$, coinciden en denominar el período entre 1870 y 1930 en el Uruguay como proceso de modernización.

e. Destacamos los trabajos de Methol Ferre ${ }^{50}$ (p. 40), Sansón ${ }^{19}$, Caetano y Geymonat ${ }^{18}$ (p. 37).

f. Dogliottri ${ }^{9}$ estudia en profundidad el proceso de institucionalización de la educación física en Uruguay.

g. En las citas a lo largo del texto, el destacado en cursivas es nuestro.

h. Entre estos estudios se destacan los trabajos de Zubillaga y Cayota ${ }^{41}$, Barrán ${ }^{42-44}$, Da Silveira ${ }^{45}$, Caetano y Geymonat ${ }^{18}$, Da Costa ${ }^{46,47}$ y Caetano $^{21}$.

i. Este movimiento plantearía sus críticas al movimiento conservador de la Iglesia de Inglaterra (High Anglican) conocido por tractarianismo o Movimiento de Oxford. Las críticas refirieron a la política económica clásica y a la estructura jerarquizada de la Iglesia de Inglaterra, así como a su corte ascético y puritano.

j. Esta promovió el estudio de cuestiones sociales y políticas a la luz de la Encarnación ${ }^{28}$ (p. 4).

k. La noción de ethos, desde una perspectiva discursiva, refiere a cierta articulación entre cuerpo y lenguaje, en particular a la inscripción del discurso en el cuerpo de los integrantes de una comunidad. También refiere a un proceso más general de adhesión/ captura/ asujetamiento de los sujetos a una determinada posición discursiva ${ }^{55}$ (p. 69).

1. Según el diccionario Webster, el término athleticism refiere a: a) un interés intenso en atletismo [athletics]; b) una entusiasta participación en atletismo; c) un celoso estímulo al atletismo. En cambio, atletismo [athletics] refiere a: a) los ejercicios físicos, deportes o torneos practicados por atletas [athletes]; b) la práctica de actividades atléticas c) los principios de las actividades y el entrenamiento atlético. A su vez, atlético [athletic] es: a) relativo a los atletas o al atletismo; b) característico o propio de un atleta: fuerte, muscular, robusto, vigoroso, ágil, activo; c) caracterizado por una complexión robusta, torso ancho, y desarrollo muscular poderoso ${ }^{51}$ (p. 138).

m. Maurice, uno de los teólogos referentes en la doctrina de la Cristiandad Muscular, dedica uno de sus textos, "The Doctrine ofsacrifice" (1854) a la ACJ de Londres ${ }^{52}$ (p. xlvixlvii).

n. "Nadie puede olvidar que la génesis y el éxito de algunos deportes - ahí está el caso del baloncesto articulado a través del YMCA- va ligado a ciertas confesiones religiosas" 54 (p. 11).

o. "La creación del significante "Cristiandad Muscular", su colocación en posición dominante, y su expansión por la red del discurso supone la tentativa de crear una nuevaposición discursiva, una nueva posición enunciativa, abriendo el espacio performativo de la (re)subjetivación" ${ }^{\prime 15}$.

p. "En la ciudad de Montevideo, a los seis días del mes de Abril del año mil novecientos nueve, reunidos en asamblea a fin de organizar la Asociación Cristiana de Jóvenes de Montevideo (...) en armonía con el propósito manifestado por el Club Protestante (...)"53. q. Bajo la presidencia de José Batlle y Ordóńez (1856-1929) se encaminaron las transformaciones que llevarían a la separación definitiva de la Iglesia Católica del Estado y a la configuración de una ciudadanía democrática laica.

r. La CNEF fue creada en 1911 (decreto-ley n. 3.798) como organismo dependiente del Poder Ejecutivo encargado de la promoción y el desarrollo de la "cultura física" a nivel nacional ${ }^{34}$ (p. 34).

s. John Mackayfue un teólogo escocés que llegó como misionero para trabajar en la ACJ de Montevideo durante el período 1925-1929. 


\section{Abstract}

Sports institutionalization and the process of secularization in Uruguay (1870-1930)

The aim of this paper is to explore the connections between the process of sports institutionalization and the process of secularization in Uruguay, in the period between 1870 and 1930, based on the relationship established between the State, the Protestant minorities and the Young Men's Christian Association (YMCA). The process of sports institutionalization in Uruguay was linked to the activities of North American Protestant missionaries who came to the region at the end of the 19th century to found the first Young Men's Christian Associations (YMCA) in South America. In 1919, Uruguay became the first secular country in South America, through the definitive separation of the state and the Catholic Church. In 1922, Montevideo was chosen by the South American YMCA Confederation as the location for its Technical Institute - where all secretaries and directors of physical education of the continent would be trained. The principle hypothesis presented in this paper, is that sport practice involved a religious program, largely unnoticed in national historic studies, which may explain the key role sports have played in the secularization process in Uruguay. The doctrine of Muscular Christianity, link to the YMCA and the protestant minorities, through sports practice, may have provided some of the elements necessary to form a new ethos or model of a secular subject in Uruguay during the period 1870-1930.This paper explores documents belonging to various private archives and to the center for physical education heritage in Uruguay (CEMEF), from a historical perspective and in light of French discursive analysis.

KeYwords: Uruguayan State; Sport; Young Men's Christian Association; Protestant minorities; Laicity.

\section{Referencias}

1. Hall DE. On the making and unmaking of monsters: Christian Socialism, muscular Christianity, and the metaphorization of class conflict. In Hall DE, editor. Muscular Christianity. Emboding the Victorian age. Cambridge: Press, Cambridge Universtiy; 2006.

2. Kidd B. Muscular Christianity and Value-centred Sport: The Legacy of Tom Brown in Canada. In Macaloon JJ, editor. Muscular Christianity in Colonial and Post-Colonial Worlds. New York: Routledge; 2009.

3. Macallon J, editor. Muscular Christianity in Colonial and Post-Colonial Worlds New York: Routledge; 2009.

4. Torres C. La educación física en Estados Unidos (1865-1945). In Scharagrodsky P, editor. La invención del "homo gymansticus. Buenos Aires: Prometeo; 2011. p. 253-277.

5. Scharagrodsky P. El scautismo en la Educación Física bonaerense o acerca del buen encauzamiento varonil (19141916). In Aisenstein Á, Scharagrodsky P. Tras las huellas de la Educación Física Escolar Argentina. Cuerpo, género y pedagogía. 1880-1950. Buenos Aires: Prometeo; 2006.

6. Da Cunha Baia A. In Associação Cristã de Moços no Brasil: um projeto de formação moral, intelectual e física (18901929).. Belo Horizonte: Universidad Federal de Minas Gerais; 2012.

7. Moctezuma L. Formando el cuerpo del ciudadano: aportes para una historia de la educación física en Latinoamérica. México: Universidad Autónoma del Estado de Morelos; 2015.

8. Da Silva GC. A Associação Cristã de Moços e experiências de escolarização da Educação Física no Brasil: sujeitos, ideias e práticas acemistas em circulação Belo Horizonte: Universidad Federal de Minas Gerais; 2017.

9. Dogliotti P. Cuerpo y curriculum: discursividades en torno a la formación de docentes de educación física en Uruguay (1874-1948).. Tesis de Maestría en Enseńanza Universitaria. Montevideo: Facultad de Humanidades y Ciencias de la Educación, UdelaR; 2012.

10. Dogliotti P. Educación del cuerpo y discursividades en torno a la formación en educación física en Uruguay (18741948) , 259 p. Comisión Sectorial de Investigación Científica U, editor. Montevideo; 2015.

11. Cabrera G, Couchet M, Dogliotti P, Malan P. La formación de directores de educación física en la etapa fundacional del Instituto Técnico de la Federación Sudamericana de Asociaciones Cristianas de Jóvenes. Revista Universitaria de la Educación Física y el Deporte. 2013;(6): p. 43-54. 
12. Craviotto A, Malan P. Entre la Gimnasia y el Deporte: la configuración de la educación física en el Uruguay (19111930). Revista Poled. 2014; 7(1).

13. Dogliotti P, Malan P. La influencia protestante y del movimiento de Springfield College en la educación del cuerpo y la educación física en el Uruguay. In Martínez Moctezuma L, editor. Formando el cuerpo del ciudadano. Aportes para una historia de la educación física en Latinoamérica. México: Universida Autónoma del Estado de Morelos; 2015.

14. Scarlato I. Corpo e tempo livre: as plazas vecinales de cultura física em MonteVidéu (1911-1915). Dissertação (mestrado). Florianópolis: Universidade Federal de Santa Catarina, Centro de Ciências da Educação, Programa de Pós-Graduação em Educação; 2015.

15. Malan P. Cristiandad Muscular y crisis del ethos valdense: Un estudio discursivo del ingreso del deporte a las Uniones Cristianas de Jóvenes de las colonias valdenses del Uruguay (1920-1970).. Tesis de conclusión de posgrado (Maestría en Educación Corporal). La Plata: Facultad de Humanidades y Ciencias de la Educación; 2017.

16. Malan P. Cristiandad Muscular y Asociación Cristiana de Jóvenes. La llegada del deporte a las colonias valdenses del Uruguay. págs. 96-121. RIDPHE-R: Revista Iberoamericana do Patrimônio Histórico-Educativo. 2018; 4(1): 96-121.

17. Malan P. Gran Bretańa, América del norte y América del Sur: el transitar de la cristiandad muscular en occidente y su llegada al Uruguay (primera mitad siglo XIX).. In En: Assbú Linhales M, Puchata Rodriguez D, Rosa MC. Diálogos transnacionais na história da educação física. Belo Horizonte: Fino Trazo; 2019. p. 169-193.

18. Caetano G, Geymonat R. La secularización en el Uruguay (1859-1919). Tomo 1. Catolicismo y privatización de lo religioso. Montevideo: Santillana; 1997.

19. Sansón T. La construcción de la nacionalidad oriental. Estudios de historiografía colonial. Udelar , editor. Montevideo; 2006.

20. Taylor M. Después de Dios. La religión y las redes de la ciencia, el arte, las finanzas y la política. Madrid: Siruela; 2011.

21. Caetano, G. El Uruguay laico. Montevideo: Taurus; 2013.

22. Elias N. Un ensayo sobre el deporte y la violencia. In Elias N, Dunning E. Deporte y ocio en el proceso de civilización. Madrid: Fondo de Cultura Económica; 1992.

23. Elias N. Introducción. In Elias N, Dunning E. Deporte y ocio en el proceso de la civilización. Madrid: Fondo de Cultura Económica; 1992.

24. Elias N. La génesis del deporte como problema sociológico. In Elias N, Dunning E. Deporte y ocio en el proceso de civilización. Madrid: Fondo de Cultura Económica; 1992.

25. Guttmann A. From Ritual to Record: the Nature of Modern Sports Nueva York: Columbia Universtiy Press; 2004.

26. Wemyss A. Histoire du Reveil:1790-1849. Paris: Les Bergers et les Mages.; 1977.

27. Putney C. Muscular Christianity. Manhood and Sports in Protestan America, 1880-1920. London: Harvard University Press; 2001.

28. Watson N, Weir S, Friend S. The development of Muscular Christianity in Victorian Britain and Beyond. Journal of Religion and Society. 2005; 7.

29. Vigarello GHR. El cuerpo cultivado: gimnastas y deportistas en el siglo XIX. In Corbin A, Jean-Jacques C, Vigarello G. Historia del Cuerpo. (II) De la Revolución Francesa a la Gran Guerra. Madrid: Taurus; 2005.

30. Monzó J. Las Asociaciones Cristianas de Jóvenes y la religión. Jóvenes. FSdACd, editor. Montevideo; 1927.

31. Pecheux M. Metáfora e interdiscurso. In Orlandi E, editor. Análise de Discurso: Michel Pêcheux. Textos selecionados. Campinas: Pontes Editores; 2011.

32. Geymonat R. Protestantismo y secularización en el Uruguay.. In Geymonat R. Las religiones en el Uruguay. Algunas aproximaciones.. Montevideo: La Gotera; 2004.

33. CNEF. Archivos de la Comisión Nacional de Educación Física. Uruguay Sport. 1922 abril;(52).

34. CNEF. La Educación Física y la Recreación en el Uruguay. Origen y Plan de Acción. Plan de Acción. Montevideo:; 1949.

35. Barran JP. Medicina y Sociedad en el Uruguay del Novecientos. Tomo 3. La invención del cuerpo Montevideo: Ediciones de la Banda Oriental; 1999.

36. Scarlato I. Plazas Vecinales de Cultura Física/Plazas de Deportes. "Templos laicos" de la ciudad batllista de Montevideo (1911-1918). Revista Iberoamericana do Patrimônio Histórico-Educativo. 2018; 4(1):62-79.

37. Hopkins J. 1912 agosto 26. Carta de Jess Hopkins al Dr. J. H. McCurdy de Springfield YMCA College.

38. Mackay JA. La filosofía del triángulo rojo.. Montevideo: Asociación Cristiana de Jóvenes; 1927.

39. Hopkins J. Quince años de Educación Física en las Asociaciones de América del Sur (1911-1926). Montevideo-Buenos Aires: Asociación Cristiana de Jóvenes; 1927. 
40. Elias N. La génesis del deporte como problema sociológico. In Elias N, Dunning E. Deporte y ocio en el proceso de civilización.. Madrid: Fondo de Cultura Económica; 1992.

41. Zubillaga C, Cayota M. Cristianos y cambio social en el Uruguay de la modernización (1896-1919) Montevideo: Ediciones de la Banda Oriental; 1988.

42. Barrán JP. Iglesia Católica y burguesía en el Uruguay de la modernización 1860-1900. Educación FdHyCdl, editor. Montevideo; 1988.

43. Barrán JP. Historia de la sensibilidad en el Uruguay. Tomo I. La cultura bárbara (1800-1860) Montevideo: Ediciones de la Banda Oriental; 1989.

44. Barrán JP. Historia de la sensibilidad en el Uruguay. Tomo II. El disciplinamiento (1860-1920). Montevideo: Ediciones de la Banda Oriental; 1990.

45. Da Silveira P. Laicidad, esa rareza. Revista Prisma. 1995;(4):154-185.

46. Da Costa N. La Laicidad Uruguaya.. Archives de sciences sociales des religions. 2009 abril-junio;(146).

47. Da Costa N. El fenómeno de la laicidad como elemento identitario. El caso uruguayo. Civitas. 2011 mayo-agosto; 11(2):207-220.

48. Malan P. La demanda planteada por la religión a la formación en Educación Física: un "ethos corporal". Revista Fermentario. 2013;(7).

49. Sansón T. La iglesia y el proceso de secularización en el Uruguay moderno (1859-1919). Hispania Sacra;63(127): 283-303.

50. Methol Ferré A. Las corrientes religiosas Montevideo: Nuestra Tierra; 1969.

51. Webster's third new international dictionary of the english language dictionary. United States of America; 1986.

52. Maurice FD. The doctrine of sacrifice. Deduced from the scriptures.A series of Sermons by Frederich Denison Maurice, M. A. Cambridge: Macmillan and Co. Cambridge; 1854.

53. ACJ. Acta fundacional de la ACJ de Montevideo, 1909; Montevideo.

54. Bentancor Ma, Vilanou C. Consideraciones histórico-antropológicas sobre el origen de la educación física y el deporte: Un ensayo taxonómico. Apunts. Educación Física y Deportes. 1995;(40):7-26.

55. Maingueneau D. Ethos, cenografia, incorporação. In Amossy R, editor. Imagens de si no discurso: a construção do ethos. São Pablo: Contexto; 2005.

\begin{tabular}{|c|c|}
\hline $\begin{array}{r}\text { ENDEREÇO } \\
\text { Paula Malan } \\
\text { Departamento de Educación Física y Prácticas Corporales } \\
\text { Instituto Superior de Educación Física } \\
\text { Universidad de la República } \\
11400 \text { - Montevideo - Uruguay } \\
\text { E-mail: paulamalan@gmail.com }\end{array}$ & $\begin{array}{l}\text { Submetido: 30/ 06/ } 2020 \\
\text { Aceito: 07/ 07/ } 2020\end{array}$ \\
\hline
\end{tabular}

